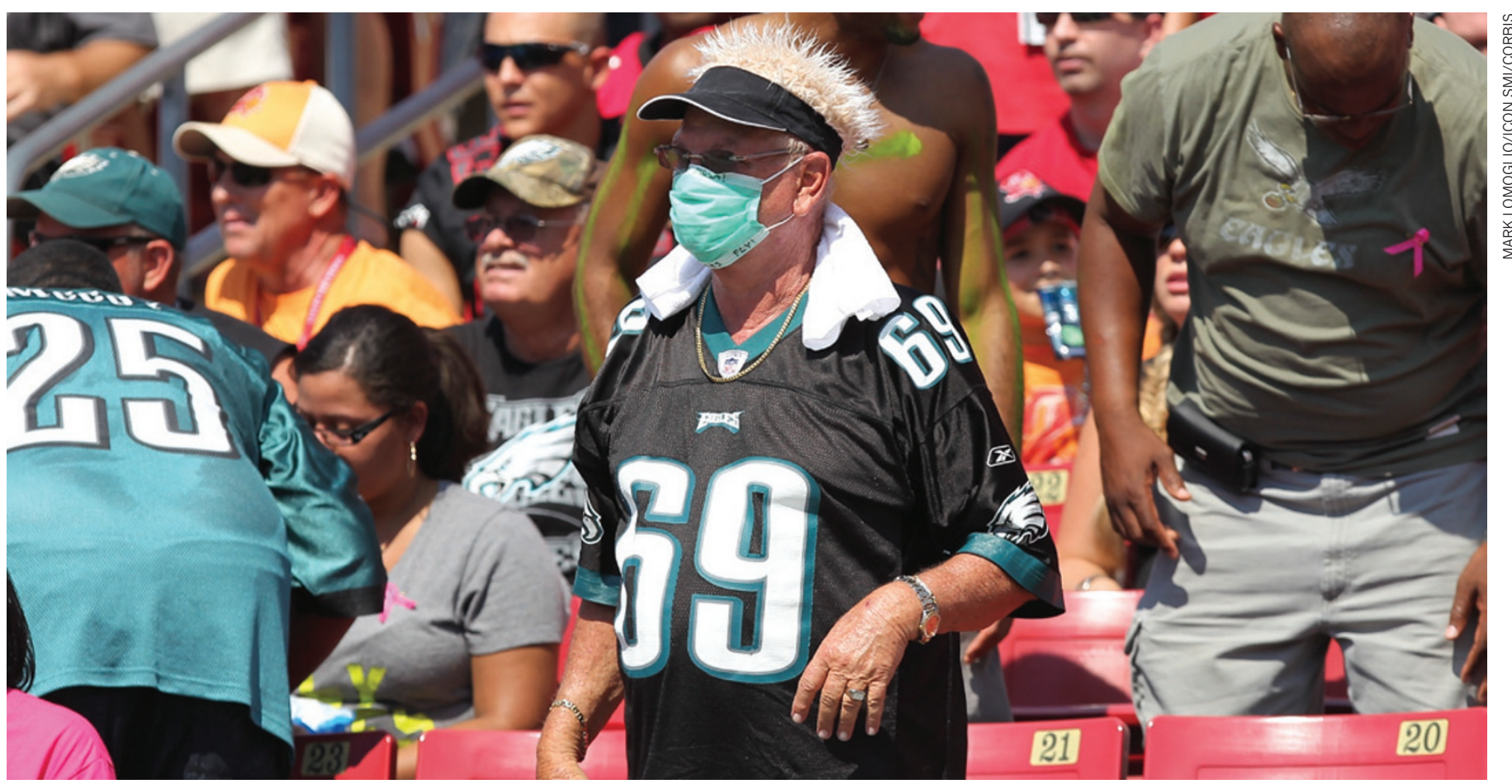

American football felt the fear of dwindling antibiotic effectiveness when methicillin-resistant Staphylococcus aureus infected the Tampa Bay Buccaneers.

\title{
Resistance fighters
}

\section{Science goes back to nature to decipher and disrupt the mechanisms by which germs evade antibiotics.}

\section{BY BILL CANNON}

( n Sunday 20 October 2013, American football team the Atlanta Falcons defeated the Tampa Bay Buccaneers. The game that day in the Georgia Dome, in the heart of downtown Atlanta, will not be remembered for its excellence - both teams finished at the bottom of their league. It was what happened after the game that hit the headlines. Another team - wearing not football uniforms but white hazardous-materials suits - entered and thoroughly disinfected the locker room used by the Buccaneers.

Back at their training facilities in Florida, several of the players had contracted methicillinresistant Staphylococcus aureus (MRSA), a drug-resistant bacteria that is becoming increasingly prevalent - the number of people admitted to hospital in the United States with the bug doubled in just five years ${ }^{1}$. Falcons' officials were worried that MRSA had hitched a ride to Atlanta on the skin of their opponents.

The concern goes beyond the Buccaneers. MRSA and other Staphylococcus infections are so common that schools and colleges in the United States are routinely watching for them. The
Chapel Hill High School in North Carolina, for instance, warns young athletes in its wrestling manual to "inform a coach immediately if they notice unusual marks on their skin", adding: "It is vital that the situation be dealt with quickly to prevent the infection of other team members." Almost everyone at the school knows someone who has been infected with MRSA.

However, our greatest risk of antibioticresistant infections may come not from fellow humans, but from cows, pigs and chickens. In 2011 , about $80 \%$ of antibiotics in the United States went to livestock, to keep them healthy in intensive breeding conditions where infections are common and can spread quickly, and to boost growth (see 'The politics of antibiotics', page S16). Living within a mile of a factory pig farm in Iowa, America's top pork-producing state, nearly triples the odds of MRSA colonization $^{2}$. In the United Kingdom, the expansion of pig farming in Staffordshire and Derbyshire has stirred fear of runaway antibiotic resistance.

In recent years, academic researchers have made leaps in understanding how microbes such as MRSA, carbapenem-resistant Enterobacteriaceae and others spread their resistance so far and wide, passing on mechanisms for evading antibiotics in a metaphorical genetic marketplace.

\section{MOBILE RESISTANCE}

Bacteria build resistance in two basic ways: vertically, by passing on mutations when a cell replicates; and horizontally, where genes are passed from one cell to another in DNA fragments called plasmids (see 'Two ways to spread'). "Plasmids are a dominant force in HGT [horizontal gene transfer]," says microbiologist Gautam Dantas of Washington University in St Louis, Missouri. "But there are important other ways in which HGT can occur, including transfer of linear parts of the chromosome from one cell to the other through the action of phages, as well as naked DNA uptake from dead cells," or DNA that is no longer encapsulated within a living bacterial cell and is free in an environment. Many researchers now consider horizontal transmission to be the primary driver of antibiotic resistance ${ }^{3}$.

"Single genes give single phenotypes," says Dantas, referring to the observable characteristics and traits of an

\section{DNATURE.COM} Find out more about the development of antibiotic resistance: go.nature.com/BrAFni 
organism. "But we should not think that antibiotic-resistance genes are on their own." Any gene can be influenced by its neighbours, and genes often interact in clusters. Some will also be more mobile - better at jumping from one bacterium to another. Dantas suggests that metagenomics approaches - sequencing everything so that "we can identify resistance genes and what genetic context they are in" - will help to elucidate mechanisms of resistance, and distinguish those genes that are particularly mobile or that might work with other resistance genes to make a bug hard to target. As he explains, "This allows us to potentially distinguish resistance genes that are not going to be part of the clinical problem from that tiny fraction of genes that probably are."

Dantas views the concept of 'genetic epistasis', in which two or more genes influence one another, as strength in numbers. "We've seen, for instance from evolutionary studies, either retrospectively or prospectively, that particular genes that code for resistance tend to cluster together," he says.

By cataloguing resistance genes and the ways in which they interact, Dantas hopes that scientists can begin to make predictions. For example, how likely is a particular resistance gene to move from an organism that causes disease to team up with one that usually does not? "What's the likelihood these genes will give a particular phenotype in a particular organism?"

\section{SWITCHING IT UP}

The topic of antibiotic overuse is not new; it is often implicated as the cause of resistance. So it seems logical that decreasing the use of antibiotics would increase antibiotic sensitivity. A few years ago at Uppsala University and Växjö Hospital in Sweden, evolutionary microbiologist Dan Andersson and collaborators tested this idea. Antibiotic use can either be reduced in general - that is, by cutting down on the total number of prescriptions or the length of treatments - or else the use of specific antibiotics can be controlled.

Andersson's team tried the second approach, letting a group of patients use one antibiotic until high levels of resistance developed, and then switching them to a different drug with the hope that resistance to the first would drop. The trial failed. "Generally, I think it's difficult to reduce resistance by reducing antibiotic consumption," Andersson says. "Over a long time, it's going to happen. But in many cases, it won't happen fast enough. For it to be useful to us from a medical standpoint, reversibility has to happen within a few years." Once resistance gets started, it is hard to stop.

The length of time it takes to reverse the problem differs between bacteria and antibiotic resistance, Andersson points out. Most mechanisms that cause antibiotic resistance have negative consequences for the germ, such as slowing its growth; this is known as a 'fitness cost ${ }^{3}$. "The cost can be very small, but it's

\section{TWO WAYS TO SPREAD}

Vertical and horizontal transfer mechanisms can spread resistance to antibiotics in bacteria.
Vertical transfer

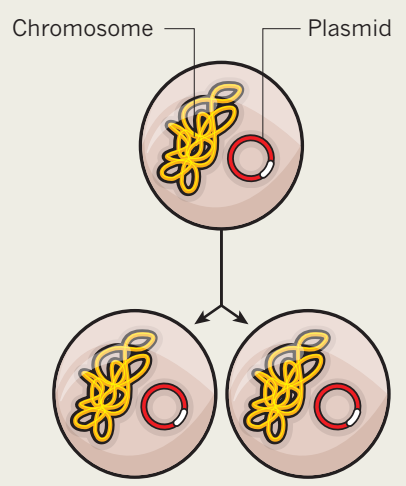

The chromosomes and plasmid are replicated during cell division and both offspring cells get complete copies.

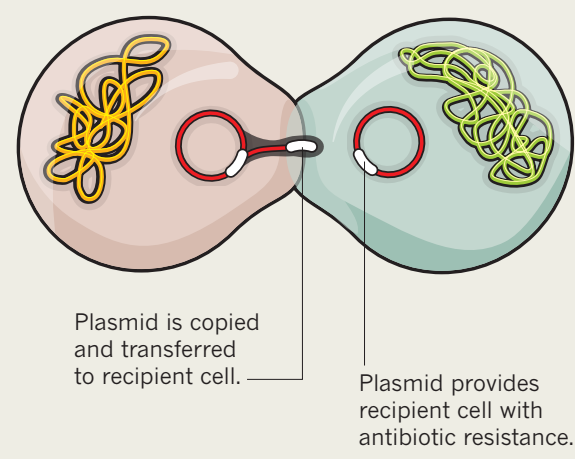

Horizontal transfer

almost always there. There are some exceptions where actually the resistant bacteria are more fit than the susceptible ones even in the absence of antibiotics, and that's really worrisome." In these cases, Andersson says, "we wouldn't expect reversibility at all because then there is no cost to drive that." When resistance raises bacterial fitness, the problem is likely to spread, even without continued use of the antibiotic.

The combination of resistance plus an increase in fitness comes from a process called compensatory evolution. Andersson gives an example: "We have looked at a lot of antibiotics that affect protein synthesis. The antibiotics bind to the ribosome; if the resistance mechanism is due to a mutation in one of the proteins in the ribosome, the compensatory mutation might be in another protein in the ribosome."

So the first mutation

"We've seen that particular genes that code for resistance tend to cluster together." gives the bacteria its resistance, but also makes it a little less fit for survival; the second mutation makes the bacteria more fit without reducing its resistance to the antibiotic. In short, the second mutation compensates for the first in terms of the bacteria's ability to survive and reproduce. The ultimate result is bacteria that are antibiotic resistant and fitter than ever.

Overall, reversing resistance is not "something we should count on as a possibility for the future", Andersson concludes. "It's not going to save us. We need new antibiotics." He adds: "The industry and academic researchers have to get into action and develop new antibiotics. But that's easier said than done."
Equally difficult would be getting physicians to stop using drugs when the frequency of resistance in a given population reaches the level at which it ceases to ascend, and doctors would still need alternative drugs. Bruce Levin, a population and evolutionary biologist at Emory University in Atlanta, Georgia, and colleagues are working on a model that shows when the resistance levels off.

In the yet-to-be-published model, Levin and microbiologist Fernando Baquero of the Ramón y Cajal Institute in Madrid, Spain, and Pål Johnsen of the University of Tromsø in Norway, who studies the evolution of bacteria, suggest that if antibiotics are used until infecting bacteria reach a target frequency of resistance - say $20 \%$ of the bacteria acquire resistance to the drug - "we can keep the level of resistance to that drug at manageable levels," Levin says. "That would work well if we had an indefinite number of drugs.” Of course, we do not. And not only is there a lack of new antibiotics, there is also the problem of multidrugresistant strains, which can be resistant to drugs that have not been used for years. Stool samples collected by Levin's team at a daycare centre showed that about $25 \%$ of the Escherichia coli bacteria isolated were resistant to streptomycin, an antibiotic that, except for tuberculosis, hasn't been used as a treatment for nearly half a century. Yet the genes for streptomycin resistance are carried on a plasmid that is resistant to commonly used antibiotics. "In too many cases, resistance appears here to stay," says Levin.

\section{COMMUNICATION BLOCK}

New antibiotics might come from a better understanding of the structural elements and communications networks of bacteria. The 
idea is to disrupt those components that lead to antibiotic resistance. A team led by Osamu Nureki, a structural biologist at the University of Tokyo in Japan, grabbed worldwide attention last year when it announced that it had solved the molecular structure of a multidrug and toxic extrusion (MATE) transporter, which confers antibiotic resistance ${ }^{5}$. In essence, the MATE transporter removes foreign things, such as toxins or antibiotics, from bacteria. While unveiling the crystal structure of the MATE transporter, Nureki's team also described a molecule that inhibits the MATE protein, the first hint of a way to block this mechanism of resistance and a possible target for drug developers (see 'Blocking an antibiotic rejection pump').

"There are lots of microbiologists trying to find bacterial Achilles' heels to make new classes of antibiotics," says molecular biologist Bonnie Bassler, a Howard Hughes Medical Institute investigator at Princeton University in New Jersey. Her lab, for example, is seeking ways to attack a bacterial communication network called quorum sensing, in which bacteria are able to determine when they are many, and in a strong position to attack. (John Woodland Hastings, a Harvard University photobiologist, first reported quorum sensing in 1970.)

In short, it works like this. Bacteria release molecules called autoinducers. If the concentration of bacteria is low, these molecules just float away. With more bacteria there are more autoinducers. "It's a concentration effect," Bassler says. "Every cell in the population is making and releasing its share of this signal molecule; the molecule increases outside the cell in proportion to cell number." She continues: "So when that molecule hits a threshold level concentration, the bacteria come into contact with those molecules and infer that they have neighbours around." The particular threshold, or 'quorum', depends on the species of bacteria.

What happens next, when pathogenic bacteria reach that quorum, can be ugly for the host. "The cells simultaneously turn on biofilm genes, virulence genes and toxins, and as a collective start releasing as a unit all of the compounds that make the host sick."

The fantasy, as Bassler bluntly describes it, is to block quorum sensing so that, even when they are crowded together, the bacteria cannot detect their neighbours and do not get the word out to build a biofilm - which is a tough-to-kill layer of bacterial cells - or to release toxins. "It looks promising," she says. "We can make molecules that are structurally similar to the real autoinducers, that jam the receptors of bacteria in a test tube, and they keep the bacteria from launching their viru"The bacteria as a result, she says, "We a collectivestart see that they can't releake biofilms; we see the that they don't kill tisthat make the host sick." sue culture cells."

Nonetheless, this remains a dream, she says, because it is uncertain whether this would work when people typically seek medical attention, after developing an established infection - and after the bacteria have realized they have a quorum and have released their coordinated, virulent onslaught. Besides that, "we need a diversity of molecules that work at different points in the quorum-sequencing pathway, to learn which place is the best therapeutically," Bassler says.

Even if those molecules were developed, they would probably need to be improved and made more potent through medicinal chemistry. Only then could they be tested as treatments in animal models and eventually humans. It is likely that we are decades away from being able to exploit quorum sensing to develop new antibiotics.

\section{DOWN TO EARTH}

Like Andersson, Dantas and others, systems biologist Roy Kishony of Technion - Israel Institute of Technology in Haifa and Harvard Medical School in Cambridge, Massachusetts, envisages drug combinations based on a deep understanding of the interplay between

\section{BLOCKING AN ANTIBIOTIC REJECTION PUMP}

Multidrug and toxic extrusion (MATE) protein removes antibiotics from bacteria, but it can be inhibited.

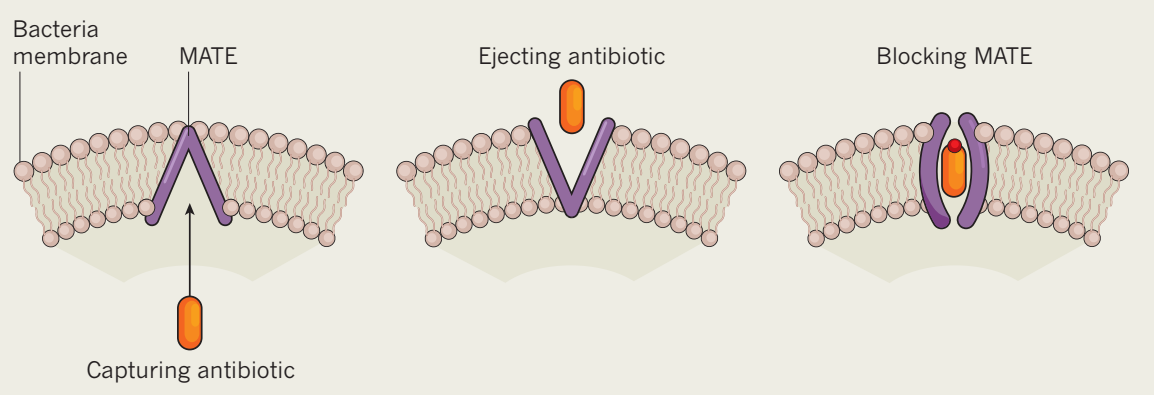

drug interactions and competition between resistance and sensitive bacteria. Kishony and colleagues compare antibiotic resistance in natural environments and the clinic. Why, they asked, is the balance between antibiotic resistance and bacterial sensitivity - that is, susceptibility to antibiotics - maintained in nature while bacterial resistance quickly takes over in the clinic ${ }^{7}$ ? Kishony points out that nature developed antibiotics hundreds of millions of years ago to curb microbes that live in the soil. "We, as the human race, are fairly new in this business, and there's much we need to learn to uncover how antibiotic resistance works in nature," he says.

In nature, antibiotics "don't exist in isolation", Kishony says. "They exist in the context of many other compounds" and in an environment where there are multiple species of bacteria. "We've taken the antibiotic out of context," he explains. "We've removed the sets of checks and balances."

Resistance, Kishony explains, usually involves only a single or a few genes. "And many of these genes have been identified and worked out to a fairly good amount of detail mechanistically and molecularly," he says.

Kishony's team pays particular attention to the mechanisms in nature that counteract resistance, to identify candidates for clinical use. "We know that while a single drug always selects for resistance, some drug combinations can actually select against bacteria becoming resistant to any one of the individual drugs." As a proof of principle, Kishony cites a study out of his lab in which drug cocktails reduced resistance across a population of doxycyclineresistant E. coli ${ }^{8}$.

He says there are likely to be many more similar mechanisms at play in the soil, which contains "an enormous number of different compounds that may interact in novel ways to inhibit the evolution of antibiotic resistance." Searching the soil for examples of these interactions may lead to new antimicrobial therapies that are more resilient to the evolution of resistance. As Kishony says, "We can invent things on our own, but we are quite likely to find great ideas and innovations just by looking in the dirt."

Bill Cannon is a freelance writer based in Chapel Hill, North Carolina.

1. David, M. Z. et al. Infect. Control Hosp. Epidemiol. 33 782-789 (2012).

2. Carrel, M. et al. Infect. Control Hosp. Epidemiol. 35, 190-193 (2014).

3. Dantas, G. \& Sommer, M. O. A. Am. Sci. 102, 42-51 (2014).

4. Andersson, D. I. \& Hughes, D. Nature Rev. Microbiol. 8, 260-271 (2010).

5. Tanaka, Y. et al. Nature 496, 247-251 (2013).

6. O'Loughlin, C. T. et al. Proc. Natl Acad. Sci. USA 110, 17981-17986 (2013).

7. Chait, R., Vetsigian, K. \& Kishony, R. Nature Chem. Biol. 8, 2-5 (2012).

8. Chait, R., Craney, A. \& Kishony, R. Nature 446 , 668-671 (2007). 